\title{
A PDMS Self-Vortical Micromixer Without Obstructions
}

\author{
Chun-You Lin*,1, Hsien-Chun Meng ${ }^{2}$ and C. $\mathrm{Fu}^{1}$ \\ ${ }^{I}$ Institute of NanoEngineering and MicroSystems, National Tsing-Hua University; ${ }^{2}$ Micro Base Technology Corp, \\ N0.101, Kuang-fu Rd., Sec.II, Hsinchu 300, Taiwan, R.O.C
}

\begin{abstract}
Microfluidic micromixers are extremely essential components of microfluidic systems for microliter to nanoliter fluid mixing. This paper proposes a new passive micromixer called the self-vortical micromixer with no obstructions, composed of three polydimethylsiloxane (PDMS) layers. Each PDMS layer is fabricated by a PDMS replication molding process with a 150-micron height dry-film photoresist replication mold, manufactured from an organic solvent-free process. The three PDMS layers are precisely bonded together by the PDMS alignment bonding process developed in this study. This paper presents the simulation results demonstrating the self-vortical mixing phenomenon of the self-vortical micromixer with swirly flow fields and self-vortical flow fields. As the mixing test results demonstrate the mixing performance of the micromixer, the injected food dyes are completely mixed in the downstream of the mixing channel by the swirly flow fields and the self-vortical flow fields in the circular mixing chamber of the self-vortical micromixer. At Re $=200$, effective mixing (mixing index 0.42) can be achieved.
\end{abstract}

Keywords: Micromixer, CFD-ACE simulation, self-vortical flow.

\section{INTRODUCTION}

Fluid mixing is an especially vital procedure in the chemical industry. Three mass transfer mechanisms, molecular diffusion, eddy diffusion, and bulk diffusion, dominate the fluid mixing phenomenon, where molecular diffusion occurs in laminar flow, and eddy diffusion and bulk diffusion occur in turbulent flow. Fluid mixing by molecular diffusion expends more time than that by eddy diffusion or bulk diffusion. The Reynolds number can indicate that fluid flow belongs to turbulent flow or laminar flow. When the Reynolds number is above 2300 , the fluid flow belongs to turbulent flow; conversely, when the Reynolds number is below 2300 , the fluid flow belongs to laminar flow. The fluid flow in the macroscopic conduits or vessels can easily generate both turbulent flow and laminar flow, yet the fluid flow in the microscopic channels or chambers can only generate laminar flow. People use turbulent flow, which is generated by a stirring magnetic bar or an ultrasonic vibration machine, to mix the milliliter volume of fluid in beakers or flasks; nevertheless, people could use only a micromixer to mix the microliter to a nanoliter volume of fluid in a microfluidic system. Micromixers are essential components used in numerous micro-chip microfluidic systems for biochemistry analysis, nucleic acid sequencing, nucleic acid synthesis, and drug discovery [1]. For example, a microfluidic biochip uses a micromixer to perform the fluidic mixing procedure of small volume biological samples and reaction reagents [2]. In microfluidic systems, the Reynolds number is below 2300 due to small container volumes and low fluid flow velocities; and the microfluid cannot generate turbulent flow. Molecular diffusion dominates the mixing efficiency, and is not efficient. Under this condition, molecular diffusion requires a long time to achieve complete mixing.

*Address correspondence to these authors at the Institute of NanoEngineering and MicroSystems, National Tsing-Hua University; N0.101, Kuang-fu Rd., Sec.II, Hsinchu 300, Taiwan, R.O.C; Tel: +886-3-571-5131 ext. 3996; Fax:+886-3-572-8023; E-mail: frankchunyoulin@gmail.com
According to the fluid mixing mechanisms of the developed micromixers in relevant literature, micromixers can be categorized as active micromixers or passive micromixers. Active micromixers mix fluids by either actuating moving parts or using external forces, such as pressure disturbances [3-5], electrical fields [6], and magnetic [7] and acoustic vibrations [8], to achieve mixing effects. Passive micromixers use complicated 3D structures and shapes along microchannels via complicated micromachining processes, and usually require mixing channels with considerable lengths and novel designs using computational fluid dynamics (CFD) simulations to achieve geometric stirring phenomena to enhance mixing effects [9-11].

This research used the circular chamber to enhance fluid mixing by using convection mixing. It is different to others design with baffles and obstruction. Their major disadvantage is induced larger flow drag or pressure loss.

\section{EXPERIMENT}

\subsection{Micromixer Design}

Using concepts of macroscopic mixers, static mixers, and Kenics mixers usually used in the chemical industry, Arnaud Bertsch et al. used a microstereolithography process to fabricate the intersecting channel micromixer $[12,13]$; Kim et al. used a microstereolithography process to fabricate the barrier-embedded Kenics micromixer [14]. This study used a simpler processes to fabricate the self-vortical micromixer, and this study designed the geometric structures using CFD simulation package software, CFD-ACE, to evaluate the mixing efficiency of the micromixer.

For two-fluid-mixing and three-fluid-mixing applications, this study designed two types of self-vortical micromixers, including the two-inlet micromixer and the threeinlet micromixer. Figs. (1a) and (b) show the schematic diagram of the microfluidic mixing principle of the two-inlet self-vortical micromixer developed in this study. In the two- 
inlet self-vortical micromixer, two fluids are separately injected into two inlet reservoirs and separately flow into two inlet channels simultaneously; the two fluids are then able to generate the self-vortical mixing phenomenon with swirly flow fields and self-vortical flow fields in the circular mixing chamber. The mixture of the two fluids is then able to flow into the mixing channel and achieve complete mixing. The two-inlet self-vortical micromixer is composed of three PDMS layers, encompassing an upper PDMS layer, a lower PDMS layer, and a bottom PDMS layer. Fig. (1c) presents the upper PDMS layer containing two inlet channels, both with a width of $500 \mu \mathrm{m}$ and a depth of $150 \mu \mathrm{m}$; one circular mixing chamber with a diameter of $2000 \mu \mathrm{m}$ and a depth of

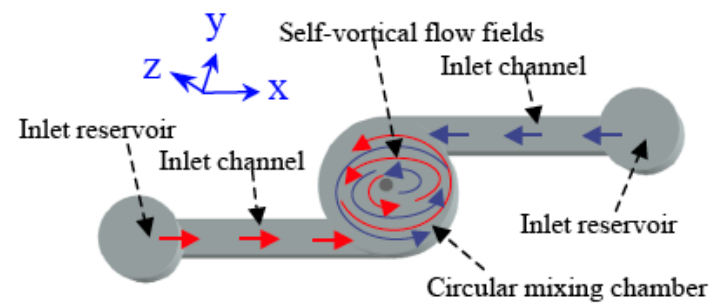

(a)

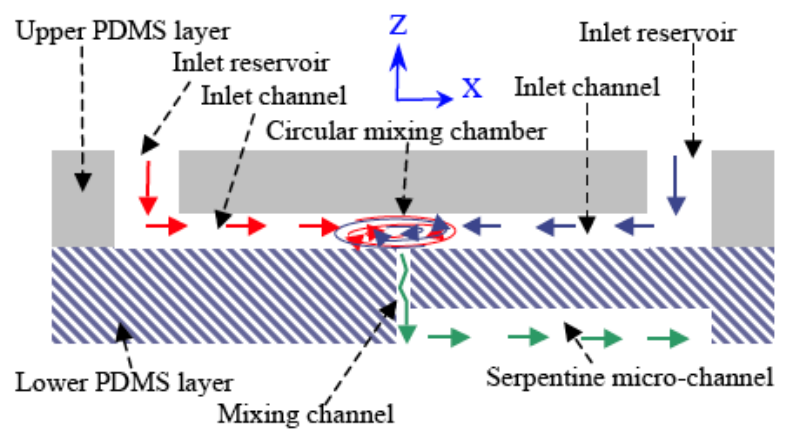

(b)

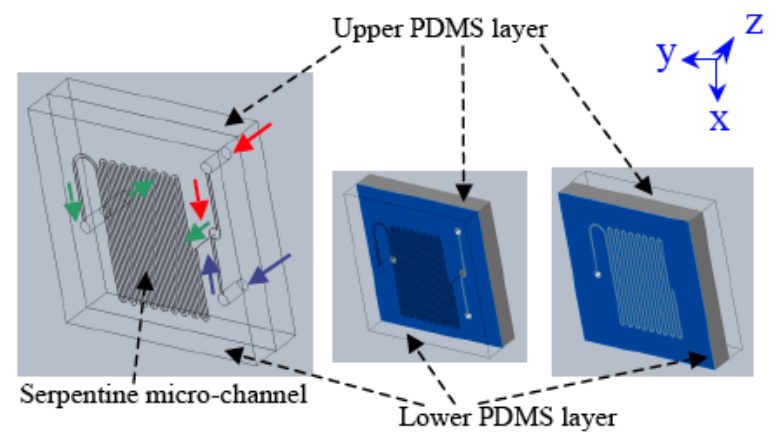

(c)

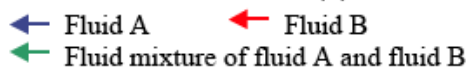

Fig. (1). The schematic diagram of the microfluidic mixing principle and design in the self-vortical micromixer. (a) Top-view of the self-vortical micromixer shows the microfluidic mixing principle. (b) Cross-section view of the self-vortical micromixer shows the microfluidic mixing principle, and (c) the designed self-vortical micromixer is composed of three PDMS layers: an upper PDMS layer, a lower PDMS layer and a bottom PDMS layer; the bottom PDMS layer is not shown here and no micro-structured patterns are apparent. The serpentine micro-channel, connected to the mixing channel, is integrated into the lower PDMS layer.
150 microns; and two inlet reservoirs, both with a diameter of $5000 \mu \mathrm{m}$ and a depth of $20 \mathrm{~mm}$. The lower PDMS layer contains one serpentine micro-channel with a width of 500 $\mu \mathrm{m}$ and a depth of $150 \mu \mathrm{m}$; one mixing channel with a width of $500 \mu \mathrm{m}$; and one outlet reservoir with a diameter of 5000 $\mu \mathrm{m}$ and a depth of $20 \mathrm{~mm}$. The bottom PDMS layer has no micro-structured patterns.

\subsection{Micromixer CFD Simulation}

The CFD simulation results can predict flow velocity profiles and flow streamlines of micromixers. This study used a commercial CFD package software, CFD-ACE+ (CFD Research Corporation), to perform micromixer designs and simulations. Fig. (2a) illustrates the self-vortical mixing phenomenon occurring in the self-vortical micromixer. Figs. (2b) and (c) show the CFD simulation results of the selfvortical micromixer with two-inlet and three-inlet, respectively. Fig. (3) shows the CFD-ACE simulation results of flow velocity profiles and flow streamlines in the circular mixing chamber at different flow rates of three inlet channels. Figs. (3a) and (b) represent the flow velocity profiles and the flow streamlines, respectively, for the inlet-fluid flow rate of $0.41 \mathrm{~mL} / \mathrm{min}(R e=25)$. Figs. (3c) and (d) represent the flow velocity profiles and flow streamlines, respectively, for the inlet-fluid flow rate of $3.30 \mathrm{~mL} / \mathrm{min}(R e=200)$. The directions of the vectors represent the directions of flow velocities, and the Re represents the Reynolds number of inlet-fluid. The CFD-ACE simulation results demonstrated that the self-vortical mixing phenomenon only occurs in the inlet-fluid flow rate under higher speed conditions.

\subsection{Micromixer Fabrication}

Polydimethylsiloxane (PDMS) polymer is a biocompatible and transparent material; and surfaces of PDMS polymer can demonstrate hydrophilic properties by oxygen plasma treatment. Polydimethylsiloxane (PDMS) with a desirable pattern can be replicated using the PDMS replication molding process. In this study, the designed self-vortical micromixer was composed of three PDMS layers, with a thickness of $20 \mathrm{~mm}$ each. Additionally, this study used the PDMS replication molding process to fabricate each PDMS layer; this study then used a PDMS-bonding process to bond the three PDMS layers together.

For the designed PDMS self-vortical micromixer, all of the depths of the patterns of the upper PDMS layer and the lower PDMS layer are 150 microns. This study should have used a 150-micron height photoresist replication mold, having the upper PDMS layer patterns and lower PDMS layer patterns, to fabricate the upper PDMS layer and lower PDMS layer using the PDMS replication molding process. A liquid type photoresist, such as SU8 (MicroChem Corporation) can be used to fabricate the 150 -micron height photoresist replication mold by a liquid-type photoresist process, but the process will produce a lot of organic solvent wastes. For reducing organic solvent wastes, this study used a dry-film photoresist to fabricate a replication mold with $150 \mu \mathrm{m}$ height dry-film photoresist using a dry-film photoresist lamination process.

For fabrication of the dry-film photoresist replication mold, this study used a roll laminating machine to perform the dry-film photoresist lamination process in five-time re- 


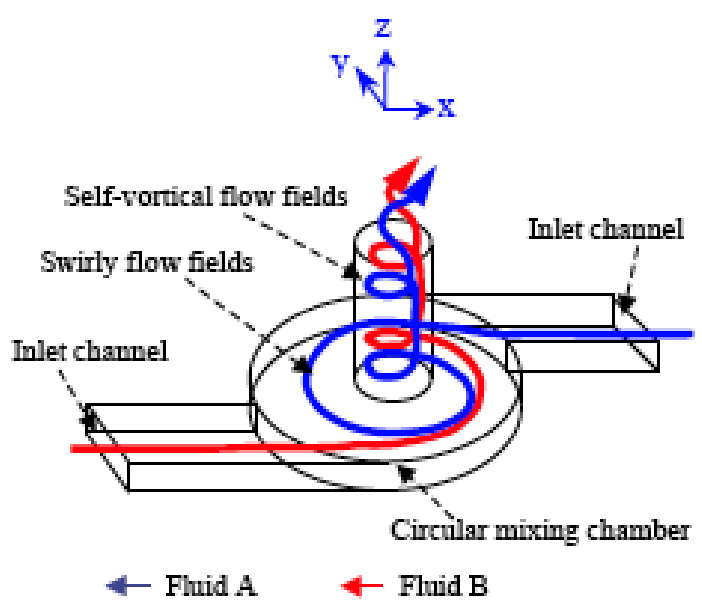

(a)

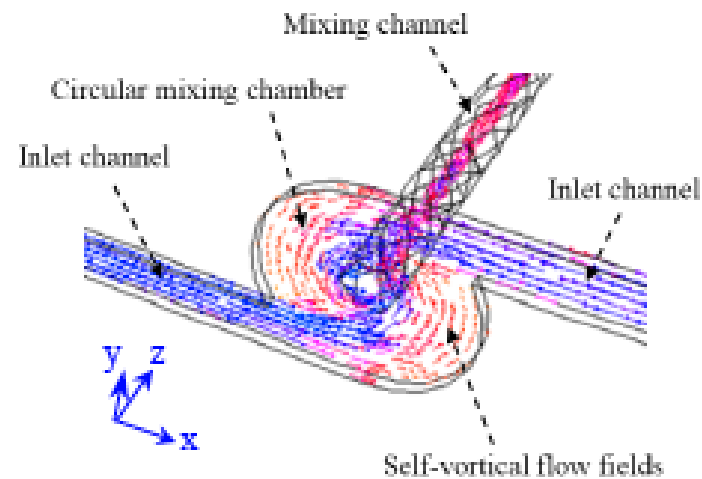

(b)

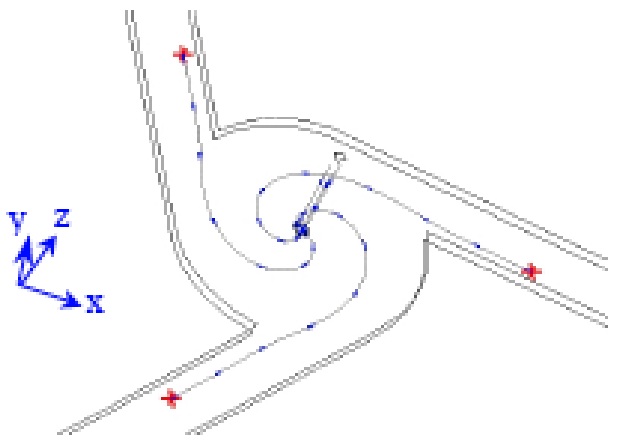

(c)

Fig. (2). The self-vortical mixing phenomenon and CFD simulation results of the self-vortical micromixer. (a) Fluid A and Fluid B separately flow into two inlet channels simultaneously, and the two fluids are then able to generate the self-vortical mixing phenomenon with the swirly flow fields and self-vortical flow fields in the circular mixing chamber. (b) and (c) represent the CFD-ACE simulation results of the two-inlet self-vortical micromixer and the three-inlet self-vortical micromixer, respectively. The directions of the vectors of the flows represent the directions of flow velocities. The profiles of the flow streamlines represent the distribution of the flow fields.

peat lamination with a $30 \mu \mathrm{m}$ thickness dry-film photoresist (Asahi Kasei Corporation) on a 4-inch glass wafer. This study then used a mask, having upper PDMS layer patterns and lower PDMS layer patterns, to perform a UV exposure process using a mask aligner (Suss MicroTec AG). Finally, this study used a water-soluble developer, a diluted sodium carbonate solution, to develop the dry-film photoresist. Fig. (4a) shows the fabrication result of the dry-film photoresist replication mold.

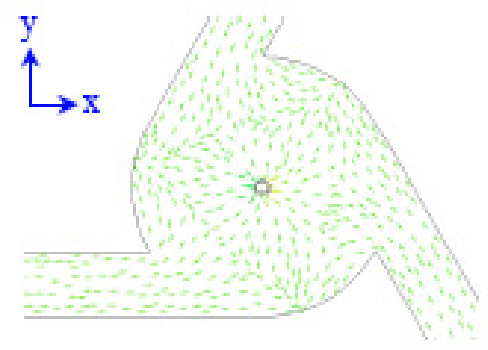

(a)

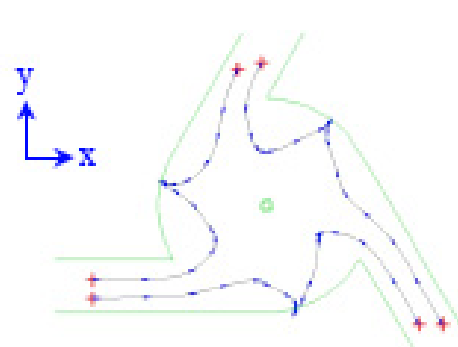

(b)

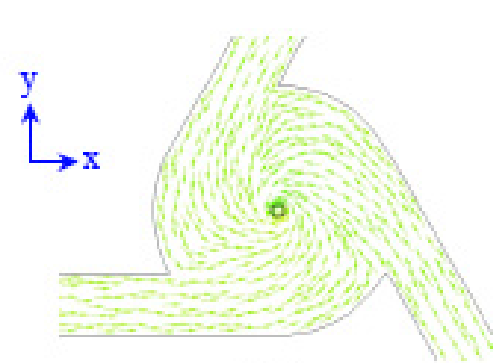

(c)

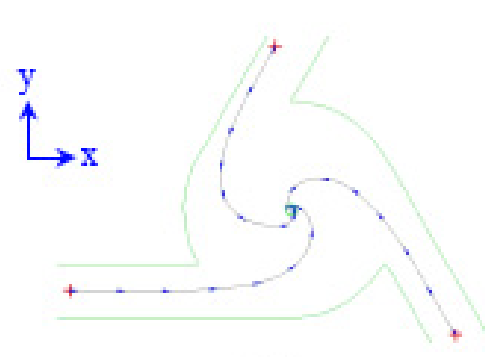

(d)

Fig. (3). The CFD-ACE simulation results of the flow velocity profiles and the flow streamlines in the circular mixing chamber at different flow rates of three inlet channels in the three-inlet selfvortical micromixer. (a) and (b) represent the flow velocity profiles and the flow streamlines, respectively, for the inlet-fluid flow rate of $0.41 \mathrm{~mL} / \mathrm{min}(R e=25)$; (c) and (d) represent the flow velocity profiles and the flow streamlines, respectively, for the inlet-fluid flow rate of $3.30 \mathrm{~mL} / \mathrm{min}(R e=200)$. The directions of the vectors represent the directions of flow velocities. $R e$ represents the Reynolds number in the inlet channels. 


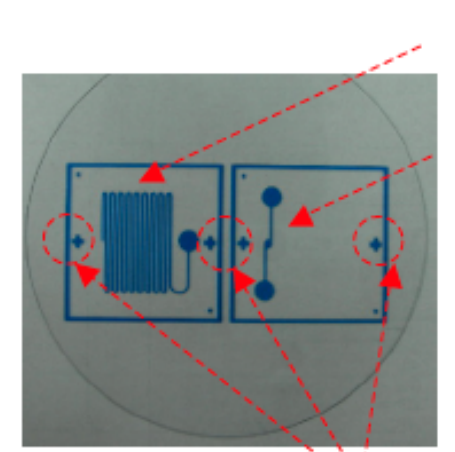

(a) Alignment keys
Dry-film photoresist with upper PDMS layer pattern

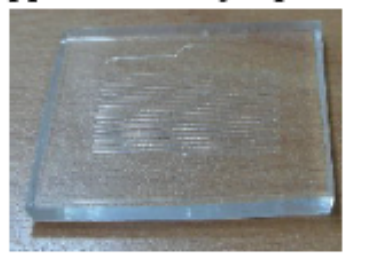

(d)

\section{lower PDMS layer pattern}

\section{Upper PDMS layer Lower PDMS layer}

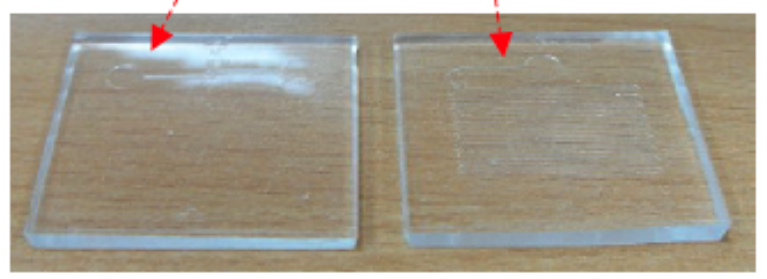

(b)

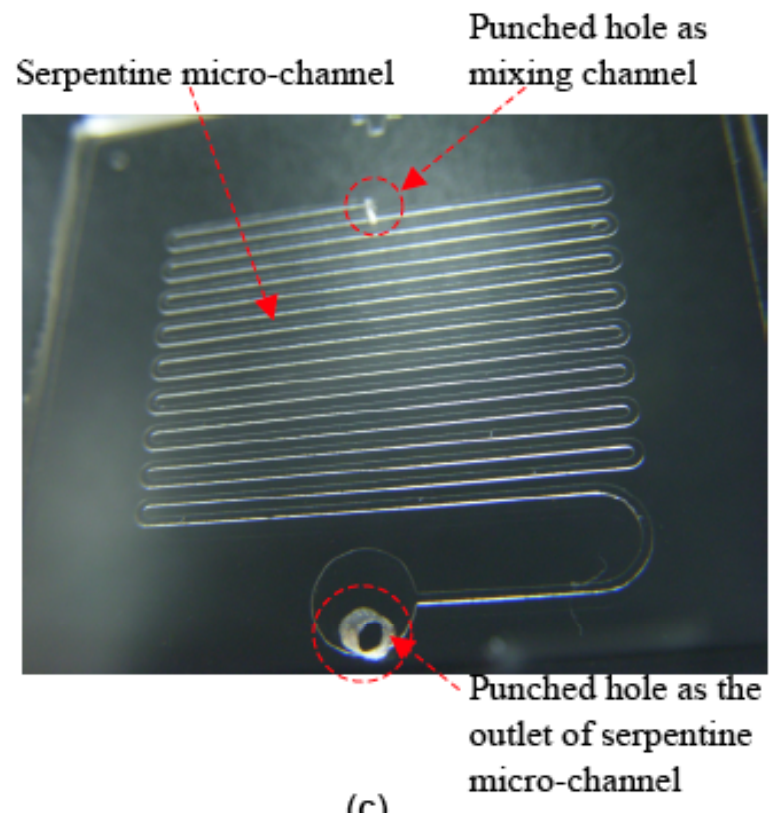

(c)

Fig. (4). The photographs of the fabrication results of the selfvortical micromixer. (a) The dry-film photoresist replication mold has the patterned dry film photoresist on 4-inch glass wafer. The patterns include the upper PDMS layer patterns, the lower PDMS layer patterns, and alignment key patterns. (b) The upper PDMS layer and lower PDMS layer were fabricated from PDMS replication molding method. (c) The magnified photograph of the lower PDMS layer shows the serpentine micro-channel and the mixing channel. (d) The upper PDMS layer and the lower PDMS layer were bonded together using bonding process.

For fabrication of the PDMS layers of the self-vortical micromixer, this study mixed a prepolymer of PDMS and a curing agent, Sylgard elastomer kit 184 (Dow Corning Co.), in a 10:1 weight-ratio and stirred thoroughly for $10 \mathrm{~min}$. The mixture was then degassed in a vacuum chamber for 1 hour.
The prepolymer mixture was then poured onto the dry-film photoresist replication mold and cured at $65^{\circ} \mathrm{C}$ for 4 hours. After curing, the molded PDMS was peeled from the dryfilm photoresist replication mold, as shown in Fig. (4b). Finally, using a puncher, this study punched the molded PDMS with several holes that served as inlets, as shown in Fig. (4c). The surface of the PDMS was activated by an oxygen plasma treatment; this study then manufactured the three PDMS layers of the self-vortical micromixer and bonded them together rapidly, as shown in Fig. (4d).

For the bonding process of the three PDMS layers of the self-vortical micromixer, this study developed a PDMS bonding tool for the three PDMS layers to bond them precisely together, as shown in Fig. (5a). This study used the characteristics suggesting that the PDMS can be bonded with a glass plate in an irreversible bond by an oxygen plasma treatment or in a reversible bond without oxygen a plasma treatment. This study also used PDMS layer alignment keys, which can be observed from a long-working distance microscope, to manufacture the three PDMS layers to be precisely bonded together using the PDMS bonding tool.

A homemade microscope with a long-working distance function was built in this study to observe the positions of alignment keys on the PDMS layers and the microfluid mixing of micromixer tests.

The homemade microscope includes a long-working distance objective (10X, M Plan Apo Series, Mitutoyo Co.), an adapter (Zoom 6000, Navitar Inc.), a high magnification zoom lens (Zoom 6000, Navitar Inc.), an illuminator, and a digital CCD camera, connected to a monitor. The upper PDMS layer of the self-vortical micromixer was mounted on the glass plate above the holder in a reversible bonding condition, and the lower PDMS layer of the self-vortical micromixer was mounted on the glass plate above a X-Y-Z translation stage in a reversible bonding condition. The X-Y$\mathrm{Z}$ translation stage can move $\mathrm{X}-\mathrm{Y}-\mathrm{Z}$ positions for alignment key observation of microscope. Both the PDMS bonding tool and the microscope were mounted on an optical table to prevent vibration.

Figs. (5b) and (c) show the results of the bonding process for the upper PDMS layer and the lower PDMS layer of the self-vortical micromixer.

\subsection{Micromixer Test}

Many types of chemicals were used in observing the microfluid mixing, including fluorescent dyes, color dyes, and $\mathrm{pH}$ indicators that were mixed in the micromixers. Fluorescent dyes include Rhodamine B [15] and a fluorescein solution [16]. Color dyes include food dyes [17] and comestible colors [18]; $\mathrm{pH}$ indicators include bromothymol blue [19] and phenolphthalein [9]. Fluorescent dyes are not favorable for re-use in the PDMS-manufactured micromixer because the PDMS becomes stained; $\mathrm{pH}$ indicators are also not favorable to be used in $\mathrm{pH}$ variational environments. This study used food dyes (yellow and blue) for high contrast and simplicity, requiring only a microscope rather than a fluorescence microscope to observe the images of the microfluid mixing. For demonstrating the mixing performance, a yellow food dye and a blue food dye were injected into inlet channels of the self-vortical micromixer using syringe pumps with a specific flow rate; the dyes then flowed through the 
mixing channel and the serpentine micro-channel in the selfvortical micromixer.

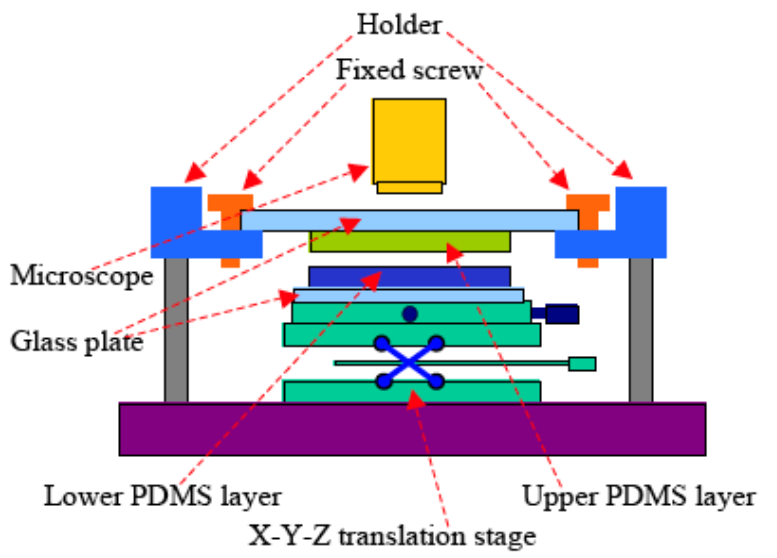

(a)

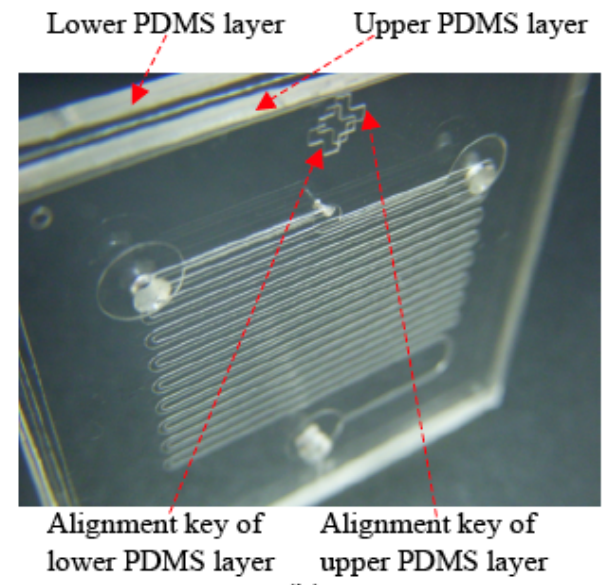

(b)

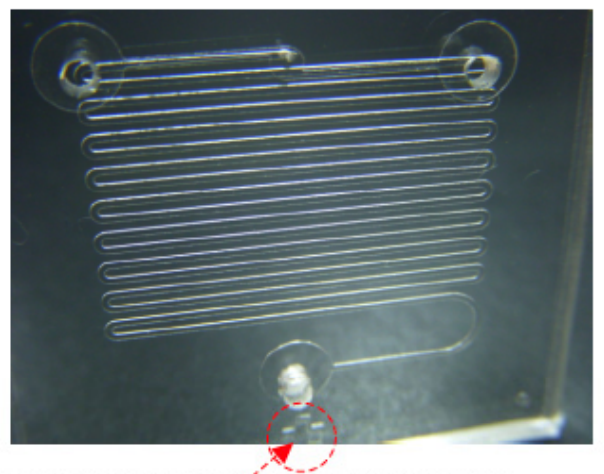

Alignment key of lower PDMS layer and alignment key of upper PDMS layer were overlapped

(c)

Fig. (5). The schematic diagram of the PDMS bonding tool and the photographs of the bonding process results for the upper PDMS layer and the lower PDMS layer of the self-vortical micromixer. (a) The X-Y-Z translation stage can move X-Y-Z positions for alignment key observation of microscope. (b) The upper PDMS layer and the lower PDMS layer were bonded together in the missing alignment condition. (c) The upper PDMS layer and the lower PDMS layer were bonded together under the perfect alignment condition.
As shown in Fig. (6), this study demonstrates the mixing test results of the two-inlet self-vortical micromixer. Simultaneously, a blue food dye fluid and a yellow food dye fluid were separately injected into two inlet channels using syringe pumps with identical flow rates of $2.4 \mathrm{~mL} / \mathrm{min}$; the dyes then flowed into the circular mixing chamber to generate the swirly flow fields and further generated the selfvortical flow fields. The blue food dye fluid and the yellow food dye fluid were mixed to become a green fluid mixture downstream of the mixing channel by the swirly flow fields and self-vortical flow fields in the circular mixing chamber of the self-vortical micromixer.

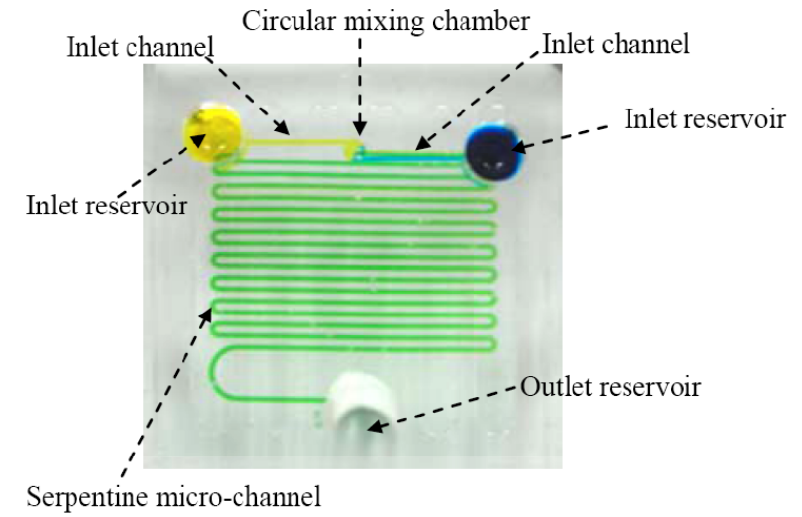

Fig. (6). The experimental result in the two-inlet self-vortical micromixer. Both blue food dye fluid and yellow food dye fluid are complete mixed to become the green fluid mixture in the downstream of the mixing channel and then flow to the serpentine microchannel.

Finally, this study quantitatively characterized the mixing efficiency of the self-vortical micromixer using the definition of the mixing index $[20,21]$. The mixing index is equals to the standard deviation of the grayscale values of the mixing image. In this study, the mixing indexes were calculated according to the grayscale values of the mixing images of the two different color dye fluids mixed at the $20 \mathrm{~mm}$ position of the downstream of the mixing channel of the self-vortical micromixer. When the blue and yellow food dye fluids are completely mixed to become a green fluid mixture, the standard deviation of the grayscale values of the mixing images is smaller than that the blue and yellow food dye fluids are not efficiently mixed. The lower mixing index indicates higher mixing efficiency. Fig. (7) shows the mixing indexes of the two different color dye fluids mixed at the different inlet-fluid flow rates in the two-inlet self-vortical micromixer. The higher inlet-fluid flow rate owns the lower mixing index, and it indicates the micromixer owns higher mixing efficiency when applying at the higher inlet-fluid flow rate.

\section{RESULTS AND DISCUSSION}

The CFD simulation results can predict the flow velocity profiles and the flow streamlines of the two-inlet selfvortical micromixer and the three-inlet self-vortical micromixer. According to the three-inlet self-vortical micromixer CFD-ACE+ simulation results, while the inlet-fluid flow rate is under a slower speed condition, such as 0.41 $\mathrm{mL} / \mathrm{min}(R e=25)$, the self-vortical mixing phenomenon does not occur in the micromixer. While the inlet-fluid flow rate is under a higher speed condition, such as $3.30 \mathrm{~mL} / \mathrm{min}$ 
$(R e=200)$, the self-vortical mixing phenomenon occurs in the micromixer. This demonstrates that the self-vortical mixing phenomenon only occurs in the inlet-fluid flow rate under a higher speed condition.

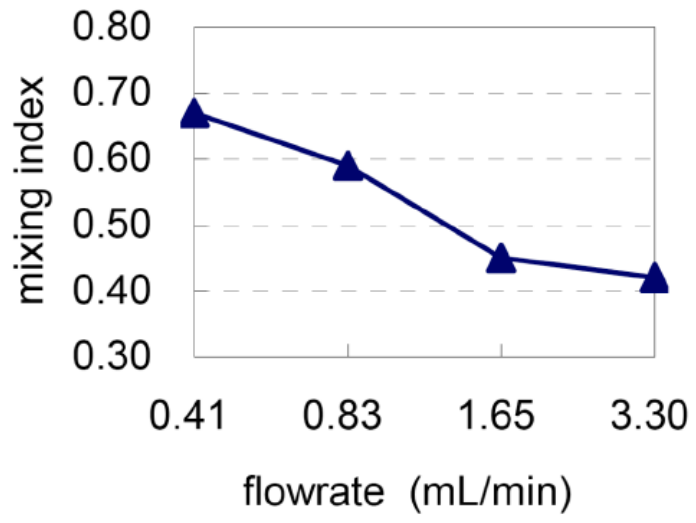

Fig. (7). The mixing indexes for the two different color dye fluids mixed at the different inlet-fluid flow rates in the two-inlet selfvortical micromixer. The lower mixing index indicates higher mixing efficiency.

According to the micromixer test results, the lower mixing index indicates higher mixing efficiency to achieve complete mixing. The blue food dye fluid and the yellow food dye fluid can be completely mixed to become a green fluid mixture downstream of the mixing channel of the selfvortical micromixer by the swirly flow fields and selfvortical flow fields.

\section{CONCLUSION}

To reduce the solvent usages in liquid-type photoresist processes, this study succeeded in applying a 30-micron thickness dry-film photoresist with an organic solvent-free dry-film photoresist lamination process to fabricate the 150micron height dry-film photoresist replication mold for use in the PDMS replication molding process. This study also developed the PDMS alignment bonding process, using PDMS layer alignment keys, as well as the characteristics of irreversible bond and reversible bond, to manufacture the three PDMS layers to be precisely bonded together using the PDMS bonding tool. Finally, this study demonstrated designs, simulations, fabrications, and mixing tests for the PDMS self-vortical micromixer, showing that the PDMS self-vortical micromixer is able to be used in a microfluidic system for fluids mixing. This design using the circular chamber will not induced large flow drag because of no any obstructions.

\section{ACKNOWLEDGEMENTS}

The authors would like to acknowledge help from Micro Base Technology Corp. (www.microbase.com.tw) and its provided facilities to test the self-vortical micromixer.

\section{REFERENCES}

[1] N.T. Nguyen, and Z. Wu, "Micromixers-a review", J. Micromech. Microeng., vol. 15, pp. R1-R16, 2005.

[2] L.C. Waters, S.C. Jacobson, N. Kroutchinina, J. Khandurina, R.S. Foote, and J.M. Ramsey, "Microchip device for cell lysis, multiplex PCR amplification, and electrophoretic sizing", Anal. Chem., vol. 70, pp. 158-162, 1998.

[3] M. Volpert, C.D. Meinhart, I. Mezic, and M. Dahelh, "An actively controlled micromixer", Proc. MEMS ASME IMECE, pp 483-487, 1999.

[4] Y.K. Lee, J. Deval, P. Tabeling, and C.M. Ho, "Chaotic mixing in electrokinetically and pressure driven micro flow", Proc. IEEE MEMS, pp 483-486, 2001.

[5] X. Niu, and Y.K. Lee, "Efficient spatial-temporal chaotic mixing in microchannels", J. Micromech. Microeng., vol. 13, pp. 454-462, 2003.

[6] M.H. Oddy, J.G. Santiago, and J.C. Mikkelsen, "Electrokinetic instability micromixing", Anal. Chem., vol. 73, pp. 5822-5832, 2001 .

[7] L.H. Lu, K.S. Ryu, and C. Liu, "A magnetic microstirrer and array for microfluidic mixing", J. Microelectromech. Syst., vol. 11, pp. 462-469, 2002.

[8] Z. Yang, H. Goto, M. Matsumoto, and R. Maeda, "Active micromixer for microfluidic systems using lead-zirconate-titanate (PZT)-generated ultrasonic vibration", Electrophoresis, vol. 21, pp. 116-119, 2000.

[9] R.H. Liu, M.A. Stremler, K.V. Sharp, M.G. Olsen, J.G. Santiago, R.J. Adrian, H. Aref, and D.J. Beebe, "Passive mixing in a threedimensional serpentine microchannel", J. Microelectromech. Syst., vol. 9, pp. 190-197, 2000.

[10] A.D. Stroock, S.K.W. Dertinger, A. Ajdari, I. Mezic, H.A. Stone, and G.M. Whitesides, "Chaotic mixer for microchannels", Science, vol. 295, pp. 647-651, 2002.

[11] C.P. Jen, C.Y. Wu, Y.C. Lin, and C.Y. Wu, "Design and simulation of the micromixer with chaotic advection in twisted microchannels", Lab. Chip, vol. 3, pp. 77-81, 2003.

[12] A. Bertsch, S. Heimgartner, P. Cousseau, and P. Renaud, "3D micromixers-downscaling large scale industrial static mixers", Proc. IEEE MEMS, pp 507-510, 2001

[13] A. Bertsch, S. Heimgartner, P. Cousseau, and P. Renaud, "Static micromixers based on large-scale industrial mixer geometry", $L a b$ Chip, vol. 1, pp. 56-60, 2001.

[14] D.S. Kim, I.H. Lee, T.H. Kwon, and D.W. Cho, "A barrier embedded Kenics micromixer", J. Micromech. Microeng., vol. 14, pp. 1294-1301, 2004.

[15] T.J. Johnson, D. Ross, and L.E. Locascio, "Rapid microfluidic mixing", Anal. Chem., vol. 74, pp. 45-51, 2002.

[16] B. He, B.J. Burke, X. Zhang, R. Zhang, F.E. Regnier, "A picolitervolume mixer for microfluidic analytical systems", Anal. Chem., vol. 73, pp. 1942-1947, 2001.

[17] L.H. Lu, K.S. Ryu, and C. Liu, "A magnetic microstirrer and array for microfluidic mixing", J. Microelectromech. Syst., vol. 11, pp. 462-469, 2002

[18] R.U. Seidel, D.Y. Si, W. Menz, and M. Esashi, "Capillary force mixing device as sampling module for chemical analysis", Proc. Trans., pp 438-441, 1999.

[19] D. Bokenkamp, A. Desai, X. Yang, Y.C. Tai, Marzluff EM, Mayo SL, "Microfabricated silicon mixers for submillisecond quenchflow analysis", Anal. Chem., vol. 70, pp. 232-236, 1998.

[20] H.Y. Wu, C.H. Liu, "A novel electrokinetic micromixer", Sensors Actuators A, vol. 118, pp. 107-115, 2005.

[21] M. Jain, K. Nandakumar, "Novel index for micromixing characterization and comparative analysis", Biomicrofluidics, 4(3): 031101, 2010 [Online]. 